BULL. AUSTRAL. MATH. SOC.

VOL. $23(1981), 191-194$.

\title{
ON A THEOREM OF BRUDNO OVER NON-ARCHIMEDIAN FIELDS
}

\author{
D. Somasundaram
}

A classical theorem of Brudno, dealing with the consistency of summability with regular matrices is shown by example not to hold over a non-archimedian field.

1.

Following Monna [1], attempts have been made in recent times to study different summability methods over non-archimedian fields which are complete in the metric of valuation. In all such attempts, as in [3], [4], significant differences in contrast to the classical case have been obtained. The object of the present short note is to prove by an example that the classical theorem of Brudno [2] dealing with the consistency of regular matrices is not true in general in the non-archimedian case. In $\S 2$, we shall describe the necessary preliminaries, where as in $\S 3$, we shall establish our claim.

2.

Let $K$ be a non-archimedian field which is complete under the metric of valuation denoted by || . We note that the valuation || is nonarchimedian if and only if $|n|<1$ for every integer $n$ considered as an element of $K$. Thus, in a field with non-trivial non-archimedian valuation, the sequence $\{1,2,3, \ldots\}=\{n\}$ is a bounded sequence in the metric of valuation.

Received 18 September 1980. 
Let $A=\left(a_{n p}\right), n, p=1,2,3, \ldots$, be a matrix defined over such a field. For $n=1,2,3, \ldots$, let us write

$$
y_{n}=\sum_{p=1}^{\infty} a_{n p} x_{p}
$$

For every sequence $x=\left\{x_{n}\right\}$ defined over $K$, let $\left\{y_{n}\right\}$ be convergent for each $n \cdot y_{n}$ is called the $A$-transform of $x$. If $y_{\dot{n}} \rightarrow y$ as $n \rightarrow \infty$ in the metric of valuation, then $x$ is said to be $A$-summable to $y$. $A$ is said to be convergence preserving if $\lim _{n \rightarrow \infty} y_{n}$ exists for every convergent sequence $x$. A is called regular if in addition $\lim _{n \rightarrow \infty} y_{n}=\lim _{n \rightarrow \infty} x_{n}$. Such regular matrices are also known as Toeplitz matrices. The theorem given below is practically contained in [1].

THEOREM (Monna). A matrix $A=\left(a_{n p}\right)$ is a regular matrix defined over $K$ if and only if $\sup _{n, p}\left|a_{n p}\right| \leq M$ where $M$ is a constant, $\lim _{n \rightarrow \infty} a_{n p}=0$ for every fixed $p, \sum_{p=1}^{\infty} a_{n p}=A_{n} \rightarrow 1$ as $n \rightarrow \infty$.

The following is the classical Brudno theorem on a regular matrix for which a simple proof was given by Petersen [2].

THEOREM (Petersen). Let every bounded sequence sumable by a Toeplitz matrix $A$ also be surmable by a Toeplitz matrix $B$. Then it is summable to the same value by $B$ as by $A$.

Petersen [2] established this theorem by showing that if two regular matrix methods $A=\left(a_{m n}\right)$ and $B=\left(b_{m n}\right)$ sum bounded sequence $\left\{s_{n}\right\}$ to different sums, then there exists a bounded sequence which is summed by $A$ but not by $B$.

3.

In this section we shall give examples of two regular matrices $A$ and $B$ over $K$ such that every bounded sequence summed by $A$ is also summed by $B$ and show that there exists a bounded sequence summable by these two regular matrices to two different sums. 
Let $A=\left(a_{n p}\right)$ and $B=\left(b_{n p}\right)$ be defined as follows:

$$
\begin{aligned}
& a_{n p}= \begin{cases}n+1 & \text { when } p=n, \\
-n & \text { when } p=n+1, \\
0 & \text { for all other values of } n \text { and } p ;\end{cases} \\
& b_{n p}= \begin{cases}n+2 & \text { when } p=n, \\
-(n+1) & \text { when } p=n+1, \\
0 & \text { for all other values of } n \text { and } p .\end{cases}
\end{aligned}
$$

The matrix $A$ satisfies the conditions of the theorem of Monna given in $\S 2$ as seen below.

(i) Since $|n+1|=\operatorname{Max}\{|n|, I\}$ and $|n|<1$, we have $|n+1|=1$. Hence we have from this $\sup _{n, p}\left|a_{n p}\right| \leq \sup \{|n+1|,|n|\}=1$.

(ii) Since each column of $A$ contains infinitely many zeros and $|n+1|=1$ and $|n|<1, a_{n p}+0$ as $n \rightarrow \infty$.

(iii) $\sum_{p=1}^{\infty} a_{n p}=n+1-n=1 \rightarrow 1$ as $n \rightarrow \infty$.

Hence $A=\left(a_{n p}\right)$ is a regular matrix. In a similar manner, we can verify that $B$ is also a regular matrix over $K$.

As a next step, we shall show that every bounded sequence summed by $A$. is also sumed by $B$. For this let $\left\{x_{n}\right\}$ be any bounded sequence. If $y_{n}$ is the A-transform of $x_{n}$, then we have $y_{n}=(n+1) x_{n}-n x_{n+1}$. If $y_{n}^{\prime}$ is the B-transform of $x_{n}$, then

$$
y_{n}^{\prime}=(n+2) x_{n}-(n+1) x_{n+1} .
$$

The relation between $y_{n}$ and $y_{n}^{\prime}$ is easily seen to be

$$
y_{n}^{\prime}=y_{n}+\left(x_{n}-x_{n+1}\right) \text {. }
$$

Hence $\left|y_{n}\right|^{\prime} \leq \operatorname{Max}\left\{\left|y_{n}\right|,\left|x_{n}-x_{n+1}\right|\right\} \leq\left|y_{n}\right|+\lambda$ where $\left|x_{n}\right| \leq \lambda$ for all $n, \lambda$ being a constant. Thus we have $\left|y_{n}^{\prime}\right| \leq\left|y_{n}\right|+\lambda$.

If $\left\{y_{n}\right\}$ is convergent, then $\left\{y_{n}^{\prime}\right\}$ is also convergent. Thus if 
$\left\{x_{n}\right\}$ is summable by $A$, then it is summable by $B$ also. This shows that the bounded convergence field of $A$ is contained in the bounded convergence field of $B$.

We establish our claim by showing that there exists a bounded sequence summable by these two regular matrices to two different sums. For this consider the bounded sequence $N=\{n\}=\{1,2,3, \ldots\}$ in $K$. The $A$-transform of the sequence $N$ gives rise to the sequence $\left(y_{n}\right)=\{0,0,0,0, \ldots\}$. So $N$ is $A$-summable to 0 . The $B$-transform of the sequence $N$ gives rise to the sequence $\left(y_{n}^{\prime}\right)=\{-1,-1,-1, \ldots\}$. So $N$ is $B$-summable to -1 . Hence given two regular methods $A$ and $B$ defined over $K$ such that every bounded sequence summed by $A$ is also summed by $B$, there exists a bounded sequence $N=\{n\}$ summable by $A$ and $B$ to two different sums which cannot happen in the case of the classical Brudno's theorem. This establishes our claim.

\section{References}

[1] A.F. Monna, "Sur le théorème de Banach-Steinhaus", Nederz. Akad. Wetensch. Proc. Ser. A 66 = Indag. Math. 25 (1963), 121-131.

[2] G.M. Petersen, "Summability methods and bounded sequences", J. London Math. Soc. 31 (1956), 324-326.

[3] D. Somasundaram, "Non-archimedian (FK)-spaces", Indian J. Math. 14 (1972), 129-140.

[4] D. Somasundaram, "Some proberties of T-matrices over non-archimedian fields", Publ. Math. Debrecen 21 (1974), 171-177.

Department of Applied Mathematics, Autonomous Post Graduate Centre, University of Madras, Coimbatore 641041 , Tamil Nadu, India. 